\title{
Forced segregation in binary granular mixtures
}

\author{
Salvatore Pillitteri ${ }^{1, *}$, Geoffroy Lumay $^{1}$, Éric Opsomer ${ }^{1}$, and Nicolas Vandewalle ${ }^{1}$ \\ ${ }^{1}$ GRASP, Institute of Physics B5a, Allée du six Août 19, University of Liège, B4000 Liège, Belgium.
}

\begin{abstract}
Mixing granular particles of different sizes is a common way of increasing the packing fraction. Recently, a model predicting the packing fraction, taking into account the inhomogeneity of the mixed small and large particles, has been proposed by S. Pillitteri et al. Under certain conditions, this model can be simplified and analytical solutions can be found. We present here these solutions, compared to experimental data, and the physical interpretation they can bring.
\end{abstract}

\section{Introduction}

Granular binary mixtures are widely studied because of the large panel of properties observed in such systems [1-8]. The main quantity describing these mixtures is the packing fraction $\eta$ which corresponds to the ratio between the true volume of the particles $V$ and the apparent volume $V_{a}$ of the pile. Depending on the relative composition of both species, the packing fraction can be strongly different.

In such systems, the control parameters are the volume fraction of small particles [8] defined as

$$
f=\frac{V_{s}}{V_{s}+V_{l}},
$$

where $V_{s}$ and $V_{l}$ are respectively the true volume of small and large particles in the mixture, and the size ratio

$$
\alpha=\frac{R_{l}}{R_{s}},
$$

where $R_{l}$ and $R_{s}$ are the radii of large and small particles. Considering the definition of $V_{s}$ and $V_{l}$, one consequently has $V=V_{s}+V_{l}$.

However, depending on the history of the granular mixture, the packing fraction can be different. Indeed, during mixing or other manipulations, segregation often occurs and leads to inhomogeneous mixtures. This inhomogeneity is known to decrease the packing fraction $[9,10]$.

In a previous article [11], we proposed a model depending on $f$ and $\alpha$ that took into account the distributions of large and small particles. In this work we propose a specific view on the simplest case, when there is a segregated monodisperse phase of large or small particles in the mixture. We show how this model can be simplified in order to obtain analytical solutions. We present these solutions and describe their effect on the packing fraction curves.

*e-mail: s.pillitteri@uliege.be

A video is available at https://doi.org/10.48448/vr1p-xg82
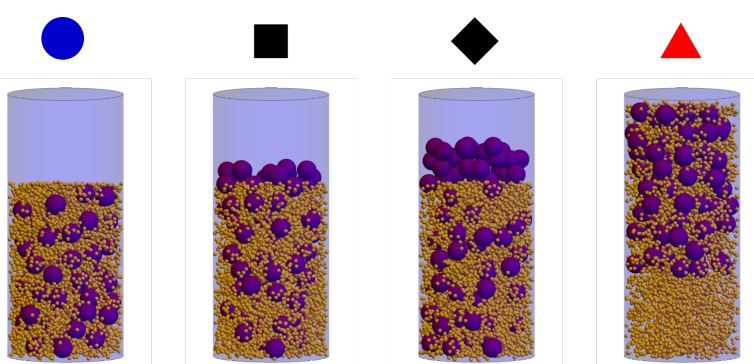

Figure 1. Examples of granular mixtures with a forced segregated part. Large particles are shown in purple while small ones are shown in orange. One observes a larger apparent volume for the segregated cases than for the homogeneous one $(\bullet)$. Large particles can be placed at the top $(\mathbf{\square}$,$) of the granular pile or small particles at the bottom$ $(\boldsymbol{\Delta})$ to force the existence of a segregated phase. Symbols are used to distinguish the different cases in Figures 2 and 3 .

\section{Method}

We prepared several mixtures by varying $f$ with a size ratio $\alpha \approx 9$ for which we forced the segregation. We defined $p$ and $b$, two segregation parameters. The parameter $p$ corresponds to the proportion of unmixed small particles which are placed at the bottom of the granular pile while $b$ represents the proportion of unmixed large particles which are placed at the top of the pile. Sketches of such mixtures are presented in Figure 1.

Forcing a partial phase separation of particles ensures a control of the segregation. For each mixture with $f, b$ and $p$ fixed, we measured the tap density curve with the GranuPack [12]. This device consists of a steel tube with a diameter $\mathrm{D}=26 \mathrm{~mm}$ and a length $\mathrm{L}=100 \mathrm{~mm}$ in which the powder is poured. A narrower tube inserted into the measurement tube is then removed in order to have a reproducible initial- 


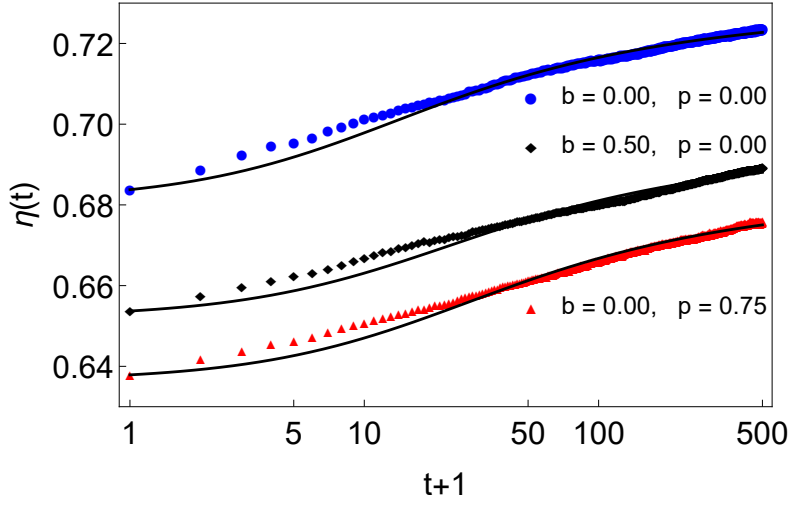

Figure 2. Three typical compaction curves for $\alpha \approx 9$ and $f=0.5$. The black curves correspond to fits using the logarithmic law eq. (3). Symbols correspond to the cases shown in Figure 1.

ization of the granular pile. The motion of the narrower tube is done at a low and constant velocity $\mathrm{v}$ $=1 \mathrm{~mm} / \mathrm{s}$. The measurement tube performs free falls or "taps" in order to densify the granular medium. The packing fraction $\eta(t)$ is recorded after each free fall until $t=500$ taps. The compaction curve is fitted with the logarithmic law [13]

$$
\eta(t)=\eta_{\infty}-\frac{\eta_{\infty}-\eta_{i}}{1+\ln (1+t / \tau)}
$$

where $\eta_{i}$ is the initial packing fraction and $\eta_{\infty}$ and $\tau$ are free fitting parameters corresponding respectively to the asymptotic packing fraction reached after the typical compaction time. We compare $\eta_{\infty}$ for the different mixtures since it corresponds to the densest packing fraction reachable by each system.

\section{Results}

In Figure 2, three typical compaction curves are presented for $\alpha \approx 9$ and $f=0.5$ for which different segregation patterns have been forced. One observes that the highest packing fraction is reached for the homogeneous case $b=p=0$. Indeed, in this case, small particles can fill voids between large pores left by large particles. On the contrary, unmixing small and large particles decreases the packing fraction. Indeed, the monodisperse phase can only reach $\eta_{\infty} \approx 0.64$, being the Random Close Packing fraction [14, 15], while the mixed part exceeds this value. The global packing fraction is a weighted average of both packing fractions. It is therefore reduced due to this unmixed part since some small particles cannot fill voids between large ones when one of the species is segregated.

Figure 3 presents the packing fraction as a function of $f$ for mixtures with unmixed parts. One observes that higher packing fractions are obtained for homogeneous mixtures. When $b \neq 0$ and $p=0$, one observes the decrease of the right part of the curve

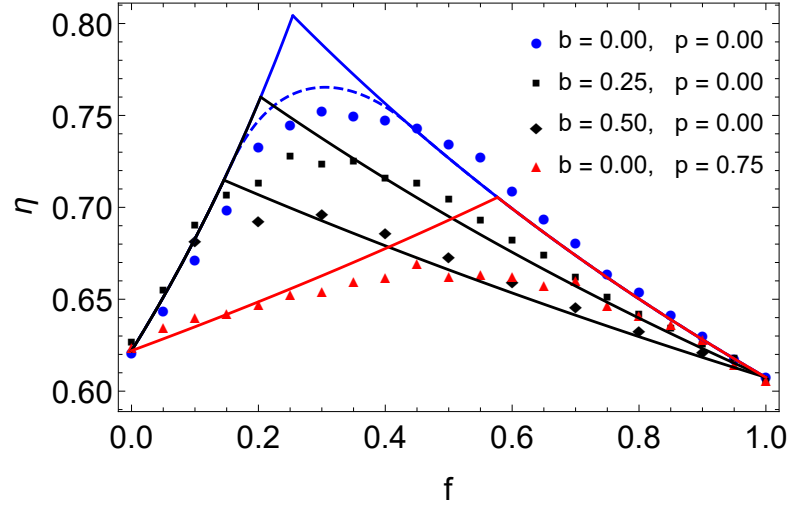

Figure 3. Asymptotic packing fraction $\eta_{\infty}$ as a function of the volume fraction of small particles $f$ for different parameters $p$ and $b$. Symbols correspond to the cases shown in Figure 1.

while the left one seems unchanged. The right part decreases as $b$ increases, as one can observe with the black curves of squares and diamonds. On the contrary the left part decreases when $p \neq 0$ and $b=0$ while the right part remains unchanged.

\section{Discussion}

This can be understood by considering the model proposed in a previous work [11]. This model stems from Furnas' approach [16] which considers both extreme cases : (I) when the packing is dominated by large particles, and (II) when the packing is dominated by small ones. Our model differs from Furnas' approach because we consider that the packing fraction should be computed by integration of a local apparent volume $V_{a}^{l o c}$ to obtain the global apparent volume $V_{a}$ when the packing is inhomogeneous. One has to define the distribution functions $\Psi_{l}(\mathbf{r})$ and $\Psi_{s}(\mathbf{r})$, respectively of the large and the small particles in the normalized unit volume $V^{*}$ of the granular pile. These functions follow the constraints

$$
\int_{V^{*}} \Psi_{l}(\mathbf{r}) \mathrm{d} r^{3}=\int_{V^{*}} \Psi_{s}(\mathbf{r}) \mathrm{d} r^{3}=1 .
$$

In the first case $(\mathrm{I})$, when $f \rightarrow 0$, the system is mainly made of large particles. One can write the local apparent volume as

$$
V_{I}^{l o c}=\left(\Psi_{l} \frac{1-f}{\eta_{l}^{0}}+\Psi_{s} \frac{f}{\eta_{s}^{0}} \frac{1}{\alpha}\right) V,
$$

where $\eta_{l}^{0}$ and $\eta_{s}^{0}$ are the packing fractions of the monodisperse cases for respectively the large and the small beads. In the second case (II), when $f \rightarrow 1$, the mixture is in a random packing configuration dominated by small beads. One can write the local apparent volume as

$$
V_{I I}^{l o c}=\left[\Psi_{s} \frac{f}{\eta_{s}^{0}}+\Psi_{l}(1-f)\left(1+\frac{1}{\alpha}\right)\right] V .
$$


The local apparent volume $V_{a}^{l o c}$ is defined as the maximum between Eqs. (5) and (6). The global apparent volume is calculated by integration of $V_{a}^{\text {loc }}$ over the unitary volume $V^{*}$. Dividing the total true volume $V$ by this global apparent volume, one has consequently for the global packing fraction

$$
\eta_{\psi}(f)=\frac{V}{\int_{V^{*}} \max \left\{V_{I}^{l o c}, V_{I I}^{l o c}\right\} \mathrm{d} r^{3}} .
$$

This model can be simplified when the system is separated into a monodisperse phase and a mixed phase as presented before. Indeed, in these peculiar conditions, the distribution functions can be approximated by a step function in the $z$ axis direction. The packing is supposed to be homogeneously distributed in the horizontal directions. One may define the function

$$
D\left(z^{*}, h^{*}, I\right)=\left\{\begin{array}{l}
\frac{I}{h^{*}} ; z^{*}<h^{*} \\
\frac{1-I}{1-h^{*}} ; z^{*} \geq h^{*}
\end{array}\right.
$$

with $z^{*} \in[0,1]$ the normalized height, $h^{*}$ the point of transition of the step-like function and $I$ the integral of this distribution between 0 and $h^{*}$. The parameter $h^{*}$ can be seen as the normalized height where the monodisperse phase and the mixed phase are neighbours and the parameter $I$ represents the proportion of particles in the range $\left[0, h^{*}\right]$. In Figure 4 are presented examples of distributions when $p \neq 0, b=0$ for (a) and $p=0, b \neq 0$ for (b). For the case (a), one consequently has $\Psi_{l}=D\left(z^{*}, h^{*}, 0\right)$ and $\Psi_{s}=D\left(z^{*}, h^{*}, p\right)$, and for the case (b) one has $\Psi_{l}=D\left(z^{*}, h^{*}, 1-b\right)$ and $\Psi_{s}=D\left(z^{*}, h^{*}, 1\right)$. One should note that the hatched area in (a) corresponds to the proportion $p$ of small particles in a monodisperse phase since $\Psi_{l}$ is nul between 0 and $h^{*}$. In case (b), the area corresponds to the proportion $b$ of large particles in a monodisperse phase.

The step function (8) is advantageous because it allows a simplification of the model. Indeed, independently of $h^{*}$, if one integrates $\max \left\{V_{I}^{\text {loc }}, V_{I I}^{\text {loc }}\right\}$ with distributions like in the case (a), the model can be reduced to

$$
\eta_{\psi}(f)=\frac{V}{\max \left\{V_{I}^{(a)}, V_{I I}^{(a)}\right\}},
$$

with

$$
V_{I}^{(a)}=\left[\frac{1-f}{\eta_{l}^{0}}+\frac{f}{\eta_{s}^{0}}\left(p+\frac{1-p}{\alpha}\right)\right] V,
$$

and

$$
V_{I I}^{(a)}=\left[\frac{f}{\eta_{s}^{0}}+(1-f)\left(1+\frac{1}{\alpha}\right)\right] V .
$$

One remarks that only $V_{I}^{(a)}$ depends on $p$. Since $\eta_{\psi}$ is a function of $\max \left\{V_{I}^{(a)}, V_{I I}^{(a)}\right\}$, this means that the peaked curves of Figure 3 correspond to a competition between $V_{I}^{(a)}$ and $V_{I I}^{(a)}$. The left part comes from $V_{I}^{(a)}$ and the right one comes from $V_{I I}^{(a)}$. One understands
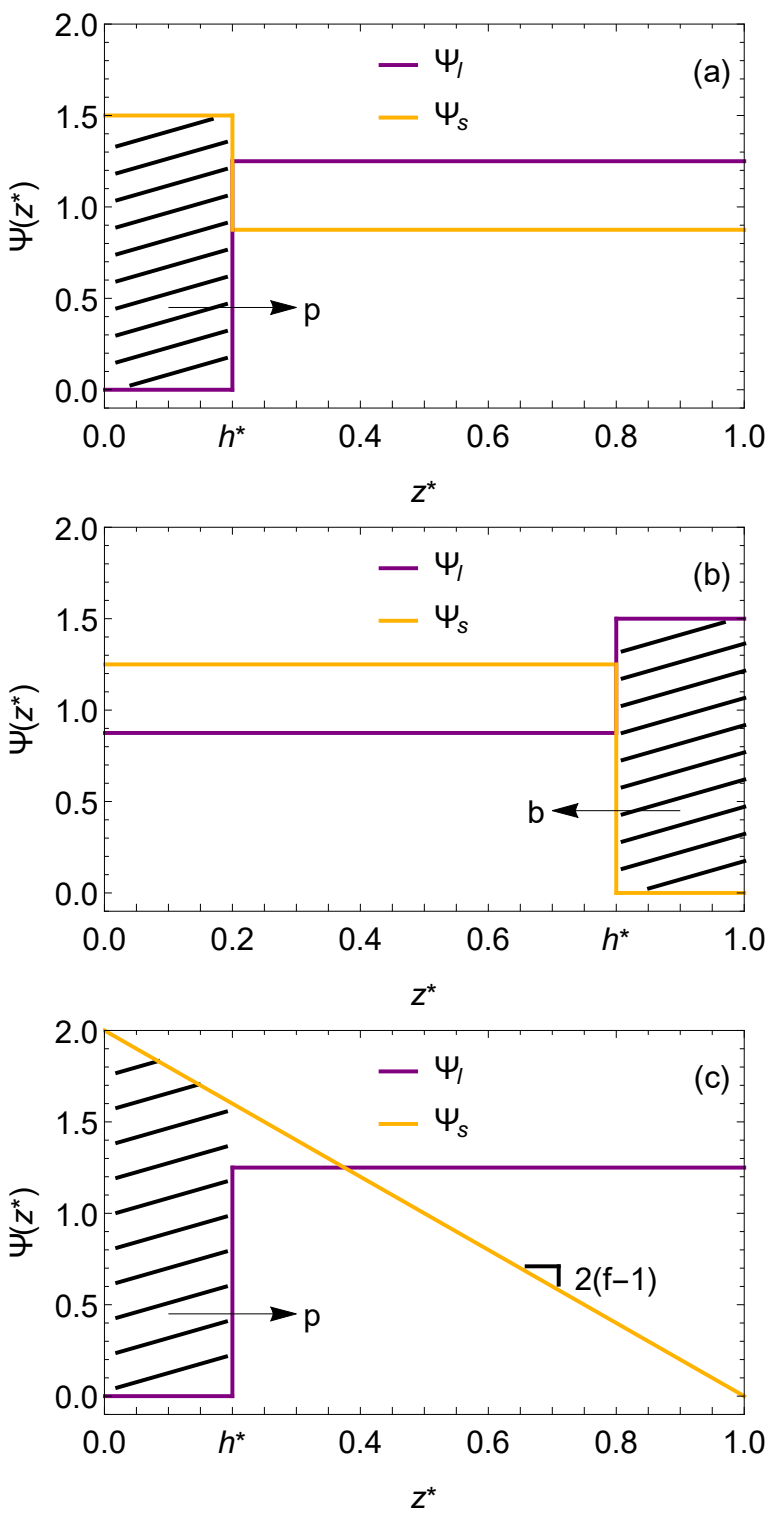

Figure 4. (a) Distributions $\Psi_{l}$ and $\Psi_{s}$ when small particles are segregated at the bottom of the granular pile. (b) Distributions $\Psi_{l}$ and $\Psi_{s}$ when large particles are segregated at the top of the granular pile. (c) Distributions $\Psi_{l}$ and $\Psi_{s}$ when small particles are distributed like a gradient with a part segregated at the bottom of the granular pile.

that since $V_{I}^{(a)}$ evolves like eq. (10), only the left part of the curve decreases when $p$ increases.

On the other hand, the case (b) gives

$$
\begin{gathered}
\eta_{\psi}(f)=\frac{V}{\max \left\{V_{I}^{(b)}, V_{I I}^{(b)}\right\}}, \\
V_{I}^{(b)}=\left(\frac{1-f}{\eta_{l}^{0}}+\frac{f}{\eta_{s}^{0}} \frac{1}{\alpha}\right) V,
\end{gathered}
$$

and

$$
V_{I I}^{(b)}=\left(\frac{f}{\eta_{s}^{0}}+\frac{1-f}{\eta_{l}^{0}}\left[b+(1-b) \eta_{l}^{0}\left(1+\frac{1}{\alpha}\right)\right]\right) V .
$$


In this case, only $V_{I I}^{(b)}$ depends on $b$, which means that only the right part decreases when $b$ increases.

One can observe the result of such modelling, knowing the fixed proportions $p$ and $b$ of respectively segregated small and large particles, in Figure 3. The model is represented by curves of the same color as the data. One can see the good agreement between the model and the experimental data.

However one can point out that the smooth optimum observed in the data is not captured by the peak shape of the model. This difference could come from the natural inhomogeneity of the mixtures. Indeed, it is highly probable that supplementary percolation occurs during manipulation. One can assume a gradient-like distribution of small particles depending on $f$ to model this inhomogeneity [11]. Indeed, when there are few small particles with $f \rightarrow 0$, one assumes that the gradient is strong. On the contrary, when $f \rightarrow 1$, their distribution should be uniform since they cannot percolate when the system is dominated by small particles. One has the distribution

$$
G\left(z^{*}, f\right)=f+2(1-f)\left(1-z^{*}\right) .
$$

The distributions $\Psi_{l}=D\left(z^{*}, h^{*}, 0\right)$ and $\Psi_{s}=$ $G\left(z^{*}, f\right)$ are presented in Figure 4 (c). In this case however, the model cannot be simplified. In addition, $h^{*}$ must increase when $f$ increases for a fixed $p$ what complicates the manipulation of the distributions. For more information about the gradient-like distribution of small particles we suggest to consult our previous article [11]. Nevertheless, we present by a dashed curve in Figure 3 the result of Eq. (7) for a mixture with $\Psi_{s}=G\left(z^{*}, f\right)$ and large particles homogeneously distributed with $\Psi_{l}=D\left(z^{*}, 0,0\right)$. Thereby, one can observe the apparition of the smooth optimum due to the gradient-like distribution of small particles.

\section{Conclusion}

We investigated the impact of partial segregation on packing fraction. We prepared mixtures with a proportion of small or large particles separated from the rest in a monodisperse phase. The decrease of the packing fraction was clearly observed as the proportion of segregated particles increases. We observed that the global packing fraction decreases in a different way if this monodisperse phase is made of large or small particles.

From these observations, we proposed here a discussion about the simplest case of our previous model [11] for inhomogeneous binary granular mixtures. Indeed, when segregation occurs by the existence of a monodisperse phase, the distributions $\Psi_{l}$ and $\Psi_{s}$ of large and small particles can be defined by step-like functions. According to the model and these distributions, one can remove the integration and simplify the expression of the apparent volume. In this way, one obtains a function $\eta_{\psi}$ which allows a direct interpretation of the decrease of the slope of the right or the left part of the curve. Depending on the nature of the monodisperse phase, made of large or small particles, the right or the left part of the curve will be affected.

We have shown that this model is adapted for the investigation of granular binary mixtures. Indeed, it takes into account the inhomogeneity of the system as well as common control parameters like the volume fraction of small particles $f$ and the size ratio $\alpha$. Since segregation often occurs in granular media, this model could be useful for granular mixtures designing and improvement. Moreover, new numerical studies about binary mixtures could be performed, with a forced gradient-like segregation, in order to test the quality of our model.

Acknowledgments - This study is conducted in the framework of the "PowderReg" project, funded by the European programme Interreg VA GR within the priority axis 4 "Strengthen the competitiveness and the attractiveness of the Grande Région / Großregion". We thank M. Marck for helping us to realize all the measurements.

\section{References}

[1] Y. Hara, H. Mizuno, A. Ikeda, (preprint) arXiv:2009.01563 (2020)

[2] I. Prasad, C. Santangelo, G. Grason, Phys. Rev. E 96, 052905 (2017)

[3] A. Tripathi, D.V. Khakhar, Phys. Fluids 23, 113302 (2011)

[4] E. Linares-Guerrero, C. Goujon, R. Zenit, J. Fluid Mech. 593, 475-504 (2007)

[5] M. Degaetano, L. Lacaze, J.C. Phillips, Eur. Phys. J. E 36, 1-9 (2013)

[6] T. Ueda, T. Matsushima, Y. Yamada, Granul. Matter 13, 731-742 (2011)

[7] L. Meng, P. Lu, S. Li, Particuology 16, 155-166 (2014)

[8] S. Pillitteri, G. Lumay, E. Opsomer, N. Vandewalle, Sci. Rep. 9, 7281 (2019)

[9] L.C. Woollacott, Miner. Eng. 131, 98-110 (2019)

[10] Z.R. Liu, W.M. Ye, Z. Zhang, Q. Wang, Y.G. Chen, Y.J. Cui, Powder Technol. 351, 92-101 (2019)

[11] S. Pillitteri, E. Opsomer, G. Lumay, N. Vandewalle, Soft Matter 16, 9094-9100 (2020)

[12] G. Lumay, F. Boschini, K. Traina, S. Bontempi, J.C. Remy, R. Cloots, N. Vandewalle, Powder Technol. 224, 19-27 (2012)

[13] J.B. Knight, C.G. Fandrich, C.N. Lau, H.M. Jaeger, S.R. Nagel, Phys. Rev. E 51, 3957 (1995)

[14] J.G. Berryman, Phys. Rev. A. 27, 1053 (1983)

[15] D. Weaire, T. Aste, The pursuit of perfect packing (CRC Press, New York, 2008)

[16] C. Furnas, Ind. Eng. Chem. 23, 1052-1058 (1931) 